DUK-M-94-05

April, 1995

\title{
Making enumerative predictions by means of mirror symmetry
}

\author{
David R. Morrison
}

\begin{abstract}
Given two Calabi-Yau threefolds which are believed to constitute a mirror pair, there are very precise predictions about the enumerative geometry of rational curves on one of the manifolds which can be made by performing calculations on the other. We review the mechanics of making these predictions, including a discussion of two conjectures which specify how the elusive "constants of integration" in the mirror map should be fixed. Such predictions can be useful for checking whether or not various conjectural constructions of mirror manifolds are producing reasonable answers.
\end{abstract}

Two-dimensional quantum field theories constructed from Calabi-Yau manifolds have been the subject of intensive study over the last several years. One of the most intriguing features of these models is the phenomenon known as mirror symmetry [28, 48, 21, 34], in which it is observed that certain pairs of Calabi-Yau manifolds produce quantum field theories which appear to be isomorphic via an automorphism which changes the sign of a certain "U(1)-charge" in the theory. This observation has provided physicists with a powerful computational tool, since calculations which are difficult in one realization of the quantum field theory may become much easier in the other (thanks to a significant shift in geometric interpretation which accompanies the sign change of the U(1)-charge). The calculations in question can often be formulated in purely mathematical terms, but it should be borne in mind that the arguments in favor of the equivalence of the answers (when the calculations are performed on two different members of a mirror pair) rely upon path integral methods which have not yet been made mathematically rigorous. For this reason, mathematicians currently regard these calculations as predicting rather than establishing the results.

The most common application of mirror symmetry as a computational tool in physics has involved calculations of certain so-called "topological" correlation functions of the physical theory. There are two types of such functions, associated to the two topological quantum field theories known as the "A-model" and the "B-model" [67], and mirror symmetry predicts that a B-model calculation on one Calabi-Yau manifold should produce the answer to a mirror A-model problem. Since that answer typically involves enumerative geometry on the mirror partner, we refer to this process as "making enumerative predictions".

Research partially supported by NSF grant DMS-9401447. 
In this paper, we review the mechanics of making these enumerative predictions (including a discussion of the reasoning which leads to the methods we describe). The starting point is a candidate mirror pair, that is, a pair of Calabi-Yau manifolds $(X, Y)$ which there is some reason to believe ought to be a pair of mirror manifolds. Precise enumerative predictions will be derived from the assumption that these manifolds are indeed a mirror pair. If those enumerative predictions can be verified, the verifications constitute evidence that a mirror partner has been correctly identified. Conversely, if discrepancies are discovered between enumerative predictions and actual enumerative calculations, the validity of either a proposed mirror construction or of the precise geometric interpretation of the quantum field theories will be called into question. In the by now rather long list of papers which have made specific enumerative predictions and attempted to verify them $[19,41,29,52,30,43,49,44,10,18$, $37,20,38,6,64]$, there have been no discrepancies (once all the dust has settled ${ }^{1}$ ).

We will restrict our attention to the enumerative problem of counting rational curves on Calabi-Yau threefolds. The very interesting extensions to higher genus [13, 14] and to higher dimension [33, 42, 39] will not be included.

The general strategy for making enumerative predictions is as follows. We start with a Calabi-Yau threefold $X$ and a candidate mirror partner $Y$ of $X$. We formulate enumerative predictions for $X$ by making Hodge-theoretic calculations on $Y$ (which are a purely mathematical version ${ }^{2}$ of the B-model calculations mentioned above). To make these predictions, we wish to compare the asymptotic expansion of the A-model correlation functions of $X$ with an appropriate expansion of B-model correlation functions of $Y$. This comparison is guided by two key observations:

1. The A-model comes with distinguished coordinates (the so-called "flat" coordinates) which correspond under mirror symmetry to coordinates on the B-model side given by ratios of periods, and

2. The coordinates on the A-model side are only well-defined up to translations by an integral lattice, which implies that the ratios of periods on the B-model side must exhibit a similar ambiguity (which will come from the monodromy of the periods).

Finding ratios of periods whose monodromy behavior (in a particular open set) reproduces the type of ambiguity expected from the A-model then identifies where the expansion of the B-model correlation function should be made.

We work in this paper with completely general Calabi-Yau threefolds, although virtually all of the specific calculations to date in the literature have dealt with special cases (complete intersections in toric varieties) which are closely related to hypergeometric functions. We

\footnotetext{
${ }^{1}$ I would like to take this opportunity to acknowledge an error in [52] (previously pointed out in [30] and [43]): the normalization in the last example of that paper was incorrect, and all entries for coefficients $n_{j}$ in the last row of table 3 of [52] should be divided by 2 .

${ }^{2}$ The connection between the physical and mathematical versions of these calculations is reviewed in detail in [23].
} 
will point out some of the special techniques which are available in those cases, although we will not review those techniques in detail.

\section{Coordinates on the B-model moduli space}

A Calabi-Yau threefold is a compact oriented 6-manifold $Y$ which admits Riemannian metrics whose (global) holonomy is contained in $\mathrm{SU}(3)$. For any such metric, there exists at least one complex structure with respect to which the metric is Kähler, and for each such complex structure $\mathcal{J}$ there is a nowhere-vanishing holomorphic 3 -form $\Omega$ on the complex manifold $Y_{\mathcal{J}}$.

Given a Calabi-Yau threefold, the topological quantum field theory known as the $B$ model of $Y$ has as its essential parameters the choice of complex structure $\mathcal{J}$ on $Y$. In fact we should identify the $B$-model moduli space with the usual moduli space of complex structures (with trivial canonical bundle) which is studied in algebraic geometry. There are some well-known technical difficulties in constructing such moduli spaces, but we are primarily concerned with two aspects of the moduli problem: we need to understand the moduli space locally, and we need to be sure that there are good compactifications of the moduli space. For Calabi-Yau manifolds, the first aspect is covered by the theorem of Bogomolov, Tian and Todorov [15, 60, 61], which says that the moduli space is smooth and that its tangent space at $\mathcal{J}$ can be naturally identified with $H^{1}\left(T_{Y_{\mathcal{J}}}^{(1,0)}\right)$, the first cohomology group of the sheaf of holomorphic vector fields. The second aspect - the existence of a good compactification - follows from Viehweg's theorem [63] that the moduli space of polarized Calabi-Yau manifolds is a quasi-projective variety. To compactify the moduli space, take a projective completion of one of Viehweg's spaces. ${ }^{3}$ Other compactifications can then be found by blowing up the original one.

The physically natural coordinates on this B-model moduli space are provided by ratios of periods of (any) holomorphic 3-form $\Omega$. That is, if $\operatorname{dim} H^{1}\left(T_{Y_{\mathcal{J}}}^{(1,0)}\right)=r$ then we choose $r+1$ elements $\gamma_{0}, \gamma_{1}, \ldots, \gamma_{r}$ in $H_{3}(Y, \mathbb{Z})$, and use the ratios $\int_{\gamma_{j}} \Omega / \int_{\gamma_{0}} \Omega$ as local coordinates. (This form of the coordinates was arrived at empirically in [19] and explained in terms of conformal field theory in [14].) At each point in the moduli space, any generic choice of such ratios will provide good local coordinates, thanks to the local Torelli theorem, the Bogomolov-TianTodorov theorem cited above, and the analysis by Bryant and Griffiths [16] of the period map for such variations of Hodge structure.

One important aspect, therefore, of the problem of making enumerative predictions will be to calculate such periods. This can sometimes be done directly, but a more common approach is an indirect one in which one first calculates the differential equations which the periods satisfy. Choose a family of holomorphic 3-forms $\Omega(s)$ which depends on a parameter $s$ on the moduli space. (This can only be done locally on the moduli space.) The periods

\footnotetext{
${ }^{3}$ Because of the polarization condition, the resulting space is only a compactification of an open subset of the original moduli space, but this is adequate for our purposes.
} 
$\int_{\gamma} \Omega(s)$ can be differentiated with respect to parameters, and there must be differential operators $\mathcal{D}$ which annihilate the periods, that is

$$
\mathcal{D}\left(\int_{\gamma} \Omega(s)\right)=0
$$

for all $\gamma \in H_{3}(Y, \mathbb{C})$. (These form a differential ideal on the moduli space.) We call these differential operators the Picard-Fuchs operators, and call the resulting differential equations $\mathcal{D} \varphi=0$ the Picard-Fuchs equations determined by $\Omega(s)$.

In principle, the Picard-Fuchs equations are derived as follows. Let $\pi: \mathcal{Y} \rightarrow S$ be a proper holomorphic map such that each fiber $\pi^{-1}(s)$ is a complex manifold diffeomorphic to $Y$ which has the complex structure corresponding to $s \in S$. (Such "universal families" should at least exist locally over the moduli space.) Let $\mathbb{V}=R^{3} \pi_{*} \mathbb{C}_{\mathcal{Y}}$ be the local system of cohomology groups, and let $\mathcal{V}=\mathbb{V} \otimes \mathcal{O}_{S}$ be the corresponding locally free sheaf, with flat connection

$$
\nabla: \mathcal{V} \rightarrow \mathcal{V} \otimes T_{S}^{*}
$$

which annihilates sections of $\mathbb{V}$. This Gauss-Manin connection can actually be computed in purely algebraic terms [40]. Doing so leads to the Picard-Fuchs equations indirectly: if we choose a basis $\gamma_{0}, \ldots, \gamma_{2 r+1}$ of $H_{3}(Y)$ then we can write

$$
\Omega(s)=\sum\left(\int_{\gamma_{j}} \Omega(s)\right) e^{j},
$$

where $e^{j}$ is the dual basis of cohomology. Then

$$
\nabla \Omega(s)=\sum\left(d \int_{\gamma_{j}} \Omega(s)\right) e^{j} .
$$

Thus, we can calculate the effect of differential operators on the periods by calculating the effect of the Gauss-Manin connection on the cohomology itself, and thereby determine the Picard-Fuchs equations.

In practice, calculating either the Gauss-Manin connection or the Picard-Fuchs equations is rather difficult. The cases in which these calculations have been carried out explicitly have involved one of two techniques:

1. In some cases it has been possible to explicitly evaluate some particular period integral, and expand its value in a power series. The Picard-Fuchs equations can then be found by finding which differential operators annihilate this known period. This method was pioneered in [19], applied in [19, 30, 43, 18, 20, 12], and reached its culmination in [11].

2. In somewhat greater generality, in many cases it has been possible to identify the periods with certain generalized hypergeometric functions; the Picard-Fuchs equations are then related to the differential equations of Gel'fand-Zelevinsky-Kapranov [31]. This method was first suggested in [8], developed in [10,3], and systematized in [37, 38]. 
We refer the interested reader to the cited papers for more details concerning this part of the calculation.

In general, the Picard-Fuchs equations will have a $(2 r+2)$-dimensional family of local solutions at any point of the moduli space, corresponding to the possible homology classes $\gamma$. The reduces the problem of identifying appropriate coordinates to the problem of selecting the "correct" homology classes $\gamma_{0}$ and other $\gamma_{j}$ 's. We address this problem in the remainder of this paper. We will identify the "correct" homology classes by comparison with the behavior of the A-model, to which we now turn.

\section{The large radius limit}

The flat coordinates in the A-model-mirror to the "ratio of periods" coordinates discussed in the previous section - have an ambiguity in their definition which can be described in terms of an integral lattice. In order to explain this, we first review some of the mathematical aspects of the moduli spaces of nonlinear $\sigma$-models (cf. $[54,56]$ ), which involve both A-model and B-model parameters.

\subsection{The nonlinear $\sigma$-model}

We briefly recall the Lagrangian formulation of nonlinear $\sigma$-models in dimension 2 . The essential ingredients needed to describe a nonlinear $\sigma$-model consist of a compact manifold $X$, a Riemannian metric $g_{i j}$ on $X$, and a class $B \in H^{2}(X, \mathbb{R} / \mathbb{Z})$, all defined up to diffeomorphisms of $X$. (We represent $B$ as a closed, $\mathbb{R} / \mathbb{Z}$-valued 2 -form, that is, as a collection of locally defined closed real 2-forms, the union of whose domains of definition is all of $X$, such that the difference between any two local representatives is $\mathbb{Z}$-valued wherever it is defined.) The nonlinear $\sigma$-model is then constructed from a $\mathbb{C} / \mathbb{Z}$-valued (Euclidean) action $\mathcal{S}$ whose bosonic part assigns to each sufficiently smooth map $\phi$ from an oriented Riemannian 2-manifold $\Sigma$ to $X$ the quantity ${ }^{4}$

$$
\mathcal{S}_{\text {bosonic }}[\phi]:=i \int_{\Sigma}\|d \phi\|^{2} d \mu+\int_{\Sigma} \phi^{*}(B)
$$

where the norm $\|d \phi\|$ of $d \phi \in \operatorname{Hom}\left(T_{\Sigma}, \phi^{*}\left(T_{X}\right)\right)$ is determined from the Riemannian metrics on $X$ and on $\Sigma$, and where $\int_{\Sigma} \phi^{*}(B)$ is a well-defined element of $\mathbb{R} / \mathbb{Z}$ by virtue of the canonical isomorphism $H^{2}(\Sigma, \mathbb{R} / \mathbb{Z}) \cong \mathbb{R} / \mathbb{Z}$. (Additional fermionic terms must be added to $\mathcal{S}_{\text {bosonic }}$ in order to make the theory supersymmetric, but as they do not affect the essential parameters in the theory we suppress them here.)

It is more customary to require $B$ to be a real 2 -form, in which case $\mathcal{S}_{\text {bosonic }}$ becomes $\mathbb{C}$-valued, and one observes that the physics is invariant under shifting $B$ by an integral

\footnotetext{
${ }^{4}$ We suppress the string coupling constant, and use a normalization in which the action appears as $\exp (2 \pi i \mathcal{S})$ in the partition and correlation functions.
} 
cohomology class. (The possibility of a more general form of the action ${ }^{5} \mathcal{S}_{\text {bosonic }}$ which allows $B$ to be an $\mathbb{R} / \mathbb{Z}$-valued 2 -form is implicit in $[62,27,6]$.) To compare this more general form to the customary one, consider the exact sequence

$$
0 \rightarrow H_{\mathrm{DR}}^{2}(X, \mathbb{Z}) \rightarrow H^{2}(X, \mathbb{R}) \rightarrow H^{2}(X, \mathbb{R} / \mathbb{Z}) \rightarrow H^{3}(X, \mathbb{Z})_{\text {tors }} \rightarrow 0,
$$

where $H_{\mathrm{DR}}^{2}(X, \mathbb{Z})$ denotes the image of $H^{2}(X, \mathbb{Z})$ in de Rham cohomology. The last term in this exact sequence is a finite group which labels the connected components of $H^{2}(X, \mathbb{R} / \mathbb{Z})$. If we only used real 2-forms modulo integral 2 -forms to describe $B$, we would get only one connected component of that space.

We are interested in a special case of this construction in which the theory has what is called $N=(2,2)$ supersymmetry and is in addition conformally invariant. To ensure the first property we assume that the Riemannian metric is Kähler with respect to some complex structure. The second property is somewhat problematic at present, but a necessary condition is that the Kähler form of the metric be in the same de Rham cohomology class as the Kähler form of some Ricci-flat metric, and that the volume of the metric be sufficiently large.

Let $\mathcal{J}$ be a complex structure on $X$ for which the metric $g_{i j}$ is Kähler. If we pick a complex structure on $\Sigma$ which makes its Riemannian metric Kähler, and which is compatible with its orientation, then the first term in the action can be rewritten using the formula:

$$
\int_{\Sigma}\|d \phi\|^{2} d \mu=\int_{\Sigma}\|\bar{\partial} \phi\|^{2} d \mu+\int_{\Sigma} \phi^{*}(\omega)
$$

where $\bar{\partial} \phi \in \operatorname{Hom}\left(T_{\Sigma}^{(1,0)}, \phi^{*}\left(T_{X_{\mathcal{J}}}^{(0,1)}\right)\right)$ is determined by the complex structures, and where $\omega$ is the Kähler form of the metric $\left(g_{i j}\right)$ on $X$. It follows that when the classical action is evaluated on a holomorphic map $\phi$ (i.e., one with $\bar{\partial} \phi \equiv 0$ ), the result is simply

$$
\int_{\Sigma} \phi^{*}(B+i \omega) \in \mathbb{C} / \mathbb{Z}
$$

The "topological" correlation functions (of both A-model and B-model type) — when evaluated using $\sigma$-model perturbation theory - depend only on these extrema of the classical action, and so ultimately will depend only on the choice of complex structure $\mathcal{J}$ and complexified Kähler form $\beta:=B+i \omega \in H^{2}(X, \mathbb{C} / \mathbb{Z})$.

\subsection{The A-model parameter space}

Not every element of $H^{2}(X, \mathbb{C} / \mathbb{Z})$ corresponds to a complexified Kähler form; the ones which do, for a fixed complex structure $\mathcal{J}$ on $X$, constitute the complexified Kähler cone

$$
\mathcal{K}_{\mathbb{C}}:=\left\{\beta \in H^{2}(X, \mathbb{C} / \mathbb{Z}) \mid \operatorname{Im}(\beta) \text { lies within the Kähler cone of } X_{\mathcal{J}}\right\} .
$$

\footnotetext{
${ }^{5}$ The more general form of the action and its properties as described in this paragraph arose in discussion with Paul Aspinwall (cf. [7]).
} 
The perturbative analysis of the $\sigma$-model is expected to be valid in some open subset of $\mathcal{K}_{\mathbb{C}}$ containing all metrics of sufficiently large volume, that is, in a set of the form

$$
\left(\mathcal{K}_{\mathbb{C}}\right)^{\circ}:=\left\{\beta \in H^{2}(X, \mathbb{C} / \mathbb{Z}) \mid \operatorname{Im}(\beta) \text { lies deep within the Kähler cone of } X_{\mathcal{J}}\right\} \text {. }
$$

The actual parameter space for $\sigma$-models with complex structure $\mathcal{J}$ can then be represented as $\left(\mathcal{K}_{\mathbb{C}}\right)^{\circ} / \operatorname{Aut}\left(X_{\mathcal{J}}\right)$, where $\operatorname{Aut}\left(X_{\mathcal{J}}\right)$ is the group ${ }^{6}$ of diffeomorphisms of $X$ which preserve the complex structure $\mathcal{J}$. We refer to this as the $A$-model parameter space, since the correlation functions of the A-model are independent of the complex structure but do depend on the parameters being described here.

A more global analysis $[2,68]$ reveals that the parameter space $\left(\mathcal{K}_{\mathbb{C}}\right)^{\circ} / \operatorname{Aut}\left(X_{\mathcal{J}}\right)$ must often be enlarged if we wish to describe the full moduli space of $N=(2,2)$ conformal field theories. But for our present purposes, we are more concerned with the "large radius limit" which occurs at the boundary of $\left(\mathcal{K}_{\mathbb{C}}\right)^{\circ} / \operatorname{Aut}\left(X_{\mathcal{J}}\right)$, and we need not worry about such enlargements.

\subsection{Flat coordinates and the large radius limit}

In order to put specific coordinates on the A-model parameter space $\left(\mathcal{K}_{\mathbb{C}}\right)^{\circ} / \operatorname{Aut}\left(X_{\mathcal{J}}\right)$, we need to choose a presentation for $H_{2}(X, \mathbb{Z})$ with generators $e_{1}, \ldots, e_{r}, f_{1}, \ldots, f_{s}$ and relations $m_{k} f_{k}=0, k=1, \ldots, s$, for some natural numbers $m_{k}>1$. Thus, $f_{1}, \ldots, f_{s}$ generate the torsion subgroup, and $e_{1}, \ldots, e_{r}$ form a basis for the free abelian group $H_{2}(X, \mathbb{Z}) /($ torsion $)$. We introduce the dual basis $e^{1}, \ldots, e^{r}$ of $H_{\mathrm{DR}}^{2}(X, \mathbb{Z})$, which will generate the integral lattice that provides the ambiguity in the flat coordinates. We make the crucial assumption that each $e^{j}$ lies in the closure of the Kähler cone.

Since $H^{2}(X, \mathbb{C} / \mathbb{Z})$ is isomorphic to $\operatorname{Hom}\left(H_{2}(X, \mathbb{Z}), \mathbb{C}^{*}\right)$, each point $\beta \in\left(\mathcal{K}_{\mathbb{C}}\right)^{\circ}$ can be regarded as a homomorphism, and as such is determined by its values on a basis, i.e., by $q_{j}:=\beta\left(e_{j}\right)$ and $\tau_{k}:=\beta\left(f_{k}\right)$, which must be nonzero complex numbers. The latter are subject to the relations $\tau_{k}^{\left(m_{k}\right)}=1$; the choice of which roots of unity to use for the $\tau_{k}$ 's determines which connected component of the parameter space we are working with. The $q_{j}$ 's are exponentials of the components of the original 2 -form, that is, when $B+i \omega \in$ $H^{2}(X, \mathbb{C}) / H_{\mathrm{DR}}^{2}(X, \mathbb{Z})$ we can write

$$
B+i \omega=\frac{1}{2 \pi i} \sum_{j}\left(\log q_{j}\right) e^{j} .
$$

It is the $\operatorname{logarithms} t_{j}:=\frac{1}{2 \pi i} \log q_{j}$ which are the "flat" coordinates. These are multiplevalued, and can be shifted by independent integers. (This indicates how the lattice $H_{\mathrm{DR}}^{2}(X, \mathbb{Z})$ specifies the ambiguity in the flat coordinates.) However, the corresponding vector fields and

\footnotetext{
${ }^{6}$ Typically, the group $\operatorname{Aut}\left(X_{\mathcal{J}}\right)$ acts discretely on $\left(\mathcal{K}_{\mathbb{C}}\right)^{\circ}$.
} 
1-forms

$$
\frac{\partial}{\partial t_{j}}=2 \pi i q_{j} \frac{\partial}{\partial q_{j}} \quad \text { and } \quad d t_{j}=\frac{1}{2 \pi i} d \log q_{j}
$$

are single-valued.

In order to study the large radius limit, we restrict our attention to those Kähler classes which lie in the cone

$$
\mathcal{C}=\mathcal{C}_{\vec{e}}:=\left\{\omega=\sum \omega_{j} e^{j} \mid \omega_{j}>0\right\}
$$

spanned by the chosen basis vectors. If the action of $\operatorname{Aut}\left(X_{\mathcal{J}}\right)$ on $\mathcal{K}_{\mathbb{C}}$ is discrete, then it will be possible to find such bases with the property that $\mathcal{C}_{\vec{e}}$ is disjoint from its translates under $\operatorname{Aut}\left(X_{\mathcal{J}}\right)$. (In any case, we shall ignore the action of $\operatorname{Aut}\left(X_{\mathcal{J}}\right)$ for the time being.) The corresponding complexified cone

$$
\mathcal{C}_{\mathbb{C}}:=\left\{\beta \in H^{2}(X, \mathbb{C} / \mathbb{Z}) \mid \operatorname{Im}(\beta) \in \mathcal{C}\right\} \subset \mathcal{K}_{\mathbb{C}}
$$

is described in coordinates by the condition

$$
\operatorname{Im}\left(\frac{1}{2 \pi i} \log q_{j}\right)=-\frac{1}{2 \pi} \log \left|q_{j}\right|>0 \text { for all } j
$$

or equivalently,

$$
0<\left|q_{j}\right|<1 \text { for all } j \text {. }
$$

To find the large radius limit, we should rescale $\omega \rightarrow \lambda \omega$, and let $\lambda$ grow to infinity. Under such a rescaling, we have

$$
q_{j} \mapsto\left|q_{j}\right|^{(\lambda-1)} q_{j}
$$

Thus, all points in $\mathcal{C}_{\mathbb{C}}$ flow towards $q_{j}=0 \forall j$ under this rescaling, and $q_{j}=0 \forall j$ should be taken as the "large radius limit." 7 We form a partial compactification of our parameter space by enlarging it to include all $q$ 's such that

$$
0 \leq\left|q_{j}\right|<1 \text { for all } j
$$

On this enlarged space, the $q_{j}$ 's occur as natural coordinates, and the "boundary" of the space is a divisor with normal crossings.

There is a natural identification which can be made between the space of marginal operators for the A-model and the vector fields $\partial / \partial t_{j}$ corresponding to the flat coordinates. When we calculate three-point functions with respect to these coordinates, we find an expansion of the form

$$
\left\langle\frac{\partial}{\partial t_{j}} \frac{\partial}{\partial t_{k}} \frac{\partial}{\partial t_{\ell}}\right\rangle=\left.e^{j} \cup e^{k} \cup e^{\ell}\right|_{[X]}+O(q),
$$

\footnotetext{
${ }^{7}$ It is not yet precisely clear how one should interpret the torsion variables $\tau_{k}$ in the large radius limit, but see [7] for some steps in this direction. For the purposes of this paper, we work with the component in which $\tau_{k}=1$ for all $k$.
} 
where $O(q)$ represents the instanton corrections to the classical value, which contain the data about the enumeration of rational curves. If we write this in terms of the (single-valued) coordinates $q_{j}$ at the large radius limit point, we find

$$
(2 \pi i)^{3} q_{j} q_{k} q_{\ell}\left\langle\frac{\partial}{\partial q_{j}} \frac{\partial}{\partial q_{k}} \frac{\partial}{\partial q_{\ell}}\right\rangle=\left.e^{j} \cup e^{k} \cup e^{\ell}\right|_{[X]}+O(q) .
$$

In other words, the three-point function has poles along the boundary divisor in the $q$ coordinates. Moreover, the leading order term in a Laurent expansion of a three-point function picks out the corresponding cohomological quantity.

The analysis we have given depends on a choice of basis; we defer to section 4 a discussion of what happens when the basis is changed.

\section{Maximally unipotent monodromy}

The structure which we have found in the A-model - a partial compactification of the parameter space, with poles of the correlation functions along the compactification divisor - will now serve as a guide to making enumerative predictions by means of B-model calculations. In order to carry this out, we must the analyze compactifications of the B-model moduli space.

Given an arbitrary compactification of the B-model moduli space, we are always free to blow up along the boundary until the boundary becomes a normal crossings divisor. The only remaining singularities of the space after such a blowup would lie in the interior of the moduli space (where there may well be quotient singularities associated with complex structures for which the automorphism group is larger than generic). Even those can be removed by passing to a finite cover.

When the boundary is a normal crossings divisor, the monodromy theorem [47] guarantees that the monodromy of the periods around each component of the boundary is a quasiunipotent transformation (unipotent after passing to a finite cover). Unipotent monodromy appears to be necessary in order to correctly reproduce the behavior of the A-model. We will therefore assume that the monodromy transformations near the points we seek are in fact unipotent.

In order to analyze the boundary in detail and search for the mirrors of large radius limit points, we restrict our attention to a local situation in which a product of punctured disks (with coordinates $s_{j}$ ) has been embedded in the interior of our moduli space in such a way that the limit points $s_{j} \rightarrow 0$ are mapped to the boundary. Let $T^{(j)}$ be the monodromy transformation about the $j^{\text {th }}$ coordinate (counterclockwise), with respect to some fixed basepoint $P$ near the origin.

For discussions of monodromy, it is more convenient to represent each period $\int_{\gamma} \Omega(s)$ by 
means of cup product with a cohomology class $g \in H^{3}\left(Y_{P}, \mathbb{C}\right)$, i.e.,

$$
\int_{\gamma} \Omega(s)=\langle g \mid \Omega(s)\rangle:=\int_{Y_{P}} g \wedge \Omega(s)
$$

The cycle $g$ extends to a multi-valued section of the local system $R^{3} \pi_{*} \mathbb{C}_{\mathcal{Y}}$, and the corresponding period is also multi-valued. However, according to the nilpotent orbit theorem [59], the section

$$
\exp \left(-\frac{1}{2 \pi i} \log s \cdot \log T\right) g \in \Gamma(\mathcal{V})
$$

of the locally free sheaf $\mathcal{V}=R^{3} \pi_{*} \mathbb{C}_{\mathcal{Y}} \otimes \mathcal{O}_{S}$ is single-valued. We introduce ${ }^{8} N^{(j)}=-\log T^{(j)}$ so that the corresponding single-valued section can be written as

$$
\widetilde{g}:=\exp \left(\frac{1}{2 \pi i} \sum\left(\log s_{j}\right) N^{(j)}\right) g
$$

We now consider the conditions on periods needed to match the behavior of the A-model. First, the period $\int_{\gamma_{0}} \Omega(s)=\left\langle g^{0} \mid \Omega(s)\right\rangle$ should be single-valued, so we need to find a cycle $g^{0}$ such that $N^{(j)} g^{0}=0$ for all $j$. Second, the monodromy on the period $\int_{\gamma_{j}} \Omega(s)=\left\langle g^{j} \mid \Omega(s)\right\rangle$ should only involve $\int_{\gamma_{0}} \Omega(s)$, in order that the multi-valuedness of the ratios shifts them by constants. Thus, we need for $N^{(j)} g^{k}$ to be a multiple of $g^{0}$ for every $j$ and $k$. In fact, if we write

$$
N^{(j)} g^{k}=m^{j k} g^{0}
$$

then the matrix $\left(m^{j k}\right)$ must be invertible in order to solve for coordinates with the desired monodromy properties. Letting $\left(m_{k \ell}\right)$ denote the inverse matrix of $\left(m^{j k}\right)$, we have

$$
\left(T^{(j)}-I\right)\left(-\sum g^{\ell} m_{\ell k}\right)=\sum N^{(j)} g^{\ell} m_{\ell k}=\delta_{k}^{j} g^{0}
$$

so this cohomology class " $-\sum g^{\ell} m_{\ell k}$ " determines the ratio of periods which has the correct monodromy properties. The corresponding (multi-valued) coordinates are then

$$
\frac{1}{2 \pi i} \log z_{k}=\frac{-1}{\left\langle g^{0} \mid \Omega\right\rangle} \sum_{\ell=1}^{r}\left\langle g^{\ell} \mid \Omega\right\rangle m_{\ell k} .
$$

As in the case of the A-model, by exponentiating these we obtain single-valued coordinates $z_{k}$ which extend across the boundary.

The coordinates as written are not uniquely specified, and in fact we have not yet used all of the information available to us by comparison with the A-model. What we have not yet considered is the three-point functions of the B-model, which should be mirror to the three-point functions of the A-model. In order to describe these, we must fix a particular choice $\Omega(s)$ of holomorphic 3 -forms on the fibers, which can be thought of as fixing the gauge

\footnotetext{
${ }^{8}$ This sign convention differs from [54], but agrees with [26].
} 
in the bundle $\pi_{*} \omega_{\mathcal{X} / S}$ whose fibers are the spaces of global holomorphic 3 -forms on the fibers of $\pi$. Moreover, as in the case of the A-model, the marginal operators whose correlation functions we wish to calculate can be identified with vector fields on the moduli space. Any system of coordinates $s_{j}$ has an associated collection of vector fields $\partial / \partial s_{j}$, and with respect to these, the three-point function can be written

$$
\left\langle\frac{\partial}{\partial s_{j}} \frac{\partial}{\partial s_{k}} \frac{\partial}{\partial s_{\ell}}\right\rangle:=\int_{Y} \Omega(s) \wedge \nabla_{s_{j}} \nabla_{s_{k}} \nabla_{s_{\ell}} \Omega(s)
$$

where $\nabla_{s_{j}} \varphi$ represents the directional derivative $\left.(\nabla \varphi)\right\lrcorner \frac{\partial}{\partial s_{j}}$.

When we calculate these three-point functions near the boundary of the moduli space, we should expect to find poles (in order to replicate the behavior of the A-model moduli space), and indeed the presence of poles in the extension of $\nabla$ to the boundary is a wellknown phenomenon in algebraic geometry (cf. [24]). These poles arise from the behavior of the single-valued sections of $\mathcal{V}$ under differentiation: if we calculate using the single-valued section $\widetilde{g}$ introduced above, we find

$$
\nabla(\widetilde{g})=\frac{1}{2 \pi i} \sum\left(d \log s_{j}\right) N^{(j)} \widetilde{g}
$$

The coefficients $d \log s_{j}$ in this expression are 1 -forms with poles along the boundary.

Taking three directional derivatives $\nabla_{s_{j}} \nabla_{s_{k}} \nabla_{s_{\ell}}$, we see that the leading term in a Laurent expansion has coefficient proportional to something of the form

$$
\left(\frac{1}{2 \pi i}\right)^{3} N^{(j)} N^{(k)} N^{(\ell)} \widetilde{g}
$$

Now the comparison with the A-model tells us that at least some of these coefficients must be nonzero, since they should reproduce the intersection numbers $\left.e^{j} \cup e^{k} \cup e^{\ell}\right|_{[X]}$ of the mirror partner. (In fact, Poincaré duality on $X$ implies some rather strong conditions on these intersection numbers, which must be replicated by the coefficients we are calculating on the B-model side.) The simple fact that any of these numbers is nonzero, though, immediately implies that the order of unipotency of the monodromy transformations is in some sense "maximal". (Any quartic expressions in the $N$ 's must vanish for dimension reasons, and so cubic expressions are the maximal possible degree for a non-vanishing expression.) Furthermore, the fact that the $N^{(j)}$ 's define a limiting mixed Hodge structure in which $h^{(3,0)}=1$ implies that the images of all of the cubic expressions lies in a one-dimensional space $W_{0}$.

Then, by including the relations deduced from Poincaré duality, we arrive at the following definition. $^{9}$

Definition A normal crossings boundary point $P$ of $S$ is called a maximally unipotent point under the following conditions.

\footnotetext{
${ }^{9}$ The original definition given in [53] only applied to the one-parameter case; this was extended to several parameters by Deligne [26] and the author [54]. (We follow the version given in [54].)
} 
1. P lies at the intersection of $r=\operatorname{dim} S$ local boundary components $B_{j}$, and the monodromy transformations $T^{(j)}$ around these components are all unipotent.

2. Let $N^{(j)}=-\log T^{(j)}$, let $N:=\sum a_{j} N^{(j)}$ for some $a_{j}>0$, and define

$$
\begin{aligned}
& W_{0}:=\operatorname{Im}\left(N^{3}\right) \\
& W_{1}:=\operatorname{Im}\left(N^{2}\right) \cap \operatorname{Ker} N \\
& W_{2}:=(\operatorname{Im}(N) \cap \operatorname{Ker}(N))+\left(\operatorname{Im}\left(N^{2}\right) \cap \operatorname{Ker}\left(N^{2}\right)\right) .
\end{aligned}
$$

Then $\operatorname{dim} W_{0}=\operatorname{dim} W_{1}=1$ and $\operatorname{dim} W_{2}=1+\operatorname{dim}(S)$.

3. Let $g^{0}, g^{1}, \ldots, g^{r}$ be a basis of $W_{2}$ such that $g^{0}$ spans $W_{0}$, and define $m^{j k}$ by $N^{(j)} g^{k}=$ $m^{j k} g^{0}$ for $1 \leq j, k \leq r$. Then $m:=\left(m^{j k}\right)$ is an invertible matrix.

(The spaces $W_{0}$ and $W_{2}$ are independent of the choice of coefficients $\left\{a_{j}\right\}[22,25]$, and the invertibility of $m$ is independent of the choice of basis $\left\{g^{k}\right\}$.)

When we restrict to maximally unipotent boundary points, the single-valued 1-forms

$$
\frac{1}{2 \pi i} d \log z_{k}=d\left(\frac{-1}{\left\langle g^{0} \mid \Omega\right\rangle} \sum_{\ell=1}^{r}\left\langle g^{\ell} \mid \Omega\right\rangle m_{\ell k}\right) .
$$

are independent of the choice of basis $\left\{g^{k}\right\}$ and of 3 -form $\Omega$. The coordinates $z_{k}$ themselves do depend on the choice of basis, but only through multiplicative constants: a change of basis replacing $g^{k}$ by $\sum_{\ell=0}^{k} c_{\ell}^{k} g^{\ell}$ will induce

$$
\frac{1}{2 \pi i} \log z_{k} \mapsto \frac{c_{0}^{k}}{c_{0}^{0}}+\frac{1}{2 \pi i} \log z_{k}
$$

and so

$$
z_{k} \mapsto e^{2 \pi i\left(c_{0}^{k} / c_{0}^{0}\right)} z_{k}
$$

Determining the "constants of integration" which specify $z_{k}$ once $d \log z_{k}$ is known is the most delicate part of finding the mirror map. We will address this issue in section 5 .

In practice, computing the monodromies about all boundary components and locating which points on the boundary have maximally unipotent monodromy is a challenging task. In fact, this computation has only been carried out fully in a few examples $[19,18,20]$. In the special cases of complete intersections in toric varieties, there is another method which has been use to locate such points: one finds the natural "toric" limit points in the toric moduli space which correspond to Kähler cones of possible birational models of the mirror, and these turn out to have maximally unipotent monodromy (as follows from $[9,12]$ ).

This alternate method must be used with some caution, for it is possible to have maximally unipotent boundary points which are not toric boundary points. An explicit example of 
this phenomon was seen in [20], where there is a non-toric boundary point with maximally unipotent monodromy. Interestingly, in that example there is also an additional (non-toric) discrete symmetry of the toric moduli space by which one must quotient in order to obtain the true B-model moduli space. That additional discrete symmetry exchanges the toric and non-toric points with maximally unipotent monodromy. It would be interesting to know whether or not this is true in general: in the toric complete intersection case, given a boundary point with maximally unipotent monodromy, does there always exist a discrete symmetry of the moduli space which maps this point to a toric boundary point?

\section{Equivalence among boundary points}

Our discussion in section 2 of coordinates near the large radius limit depended on the choice of basis for $H_{2}(X, \mathbb{Z}) /($ torsion), or equivalently, on the choice of simplicial cone $\mathcal{C} \subset \mathcal{K}$. The fact that different choices of simplicial cone (always contained in the Kähler cone) lead to apparently different "large radius limit" points in the A-model parameter space should not be too surprising. The limit point we seek is actually a boundary point of our parameter space, and what we are finding is that there are different ways to compactify the space. Since we are using compactifications which (locally) have the structure of an algebraic variety, we should expect birational modifications along the boundary to provide a mechanism for passing between compactifications and indeed that is what happens with our choice of cones. Subdividing a given cone into smaller ones precisely corresponds to blowing up, as in toric geometry.

For example, if we start from a basis $e^{1}, \ldots, e^{r}$ and blowup the origin in the coordinate chart $\left(q^{1}, \ldots, q^{r}\right)$, we find new coordinate charts after the blowup, with coordinates $\left(q_{1}, \frac{q_{2}}{q_{1}}, \ldots, \frac{q_{r}}{q_{1}}\right), \ldots,\left(\frac{q_{1}}{q_{r}}, \ldots, \frac{q_{r-1}}{q_{r}}, q_{r}\right)$, respectively. (The corresponding bases are $\left\{e^{1}+\cdots+\right.$ $\left.e^{r}, e^{2}, \ldots, e^{r}\right\}, \ldots,\left\{e^{1}, \ldots, e^{r-1}, e^{1}+\cdots+e^{r}\right\}$, respectively.) Rescaling the metric and taking $\lambda \rightarrow \infty$ sends $\left(q_{1}, \ldots, q_{r}\right)$ to the origin in the first chart when $1>\left|q_{1}\right|>\left|q_{j}\right|(\forall j \neq 1)$, sends it to the origin in the second chart when $1>\left|q_{2}\right|>\left|q_{j}\right|(\forall j \neq 2)$, and so on. All of these "origins" can thus lay claim to being "the large radius limit" associated to at least part of the A-model parameter space.

Conversely, if we have a partial compactification of the A-model parameter space which includes more than one large radius limit point (each associated with a different basis $e^{1}, \ldots, e^{r}$, and with a different domain inside the moduli space), we should attempt to blow down this space to produce a partial compactification with a single large radius limit point for the entire moduli space. These blowdowns are similar to those arising in toric geometry, and will often lead to singularities in the compactified space. The instanton contributions to correlation functions are still suppressed in such a limit, in spite of the singularities - we must accept the possibility that the "true" large radius limit point is not a smooth point.

(Note that all of the large radius limit points under discussion are associated to a single Kähler cone. It is also possible to consider other large radius limit points associated to the 
Kähler cones of different birational models of $X$. This leads to topology-changing transitions [2], and we would not expect to collapse those limit points to a single point by blowing down.)

Comparison between different cones can be accomplished by considering the canonical 1 -forms $d \log q_{j}$. These are intrinsically defined, and should only change by a constant changeof-basis matrix when moving from one large radius limit point to another (within the same Kähler cone). These 1-forms will therefore define a local system $\mathcal{L}$ in a neighborhood of all of the exceptional divisors of a potential blowing-down map associated to the Kähler cone.

We are thus led to introduce an equivalence relation among boundary points of the A-model parameter space: two boundary points $P$ and $Q$ are equivalent if there exists a connected subset $\Xi$ of the boundary containing both points and a local system $\mathcal{L}$ defined in a neighborhood of $\Xi$ which is spanned by the canonical 1-forms $d \log q_{j}$ at any maximally unipotent point within $\Xi$. (For further details about this construction, we refer the reader to [54].)

Even when we expect to be able to blow down and are willing to allow singularities, it may prove to be impossible to perform the desired blowing down, due to the presence of an infinite number of large radius limit points. We describe this phenomenon in an explicit example, following [55]. Suppose that the Kähler cone is described as $\frac{2}{1-\sqrt{5}} y<x<\frac{2}{1+\sqrt{5}} y$. Then (as shown in figure 1) attempting to cover the cone using integral bases leads to a sequence of rays with slopes $\frac{y}{x}=\ldots,-\frac{5}{8},-\frac{2}{3},-1, \frac{1}{0}, 2, \frac{5}{3}, \frac{13}{8}, \ldots$ which asymptotically approach the walls ${ }^{10}$ of the cone. Each adjacent pair of rays in the sequence gives rise to a distinct large radius limit point.

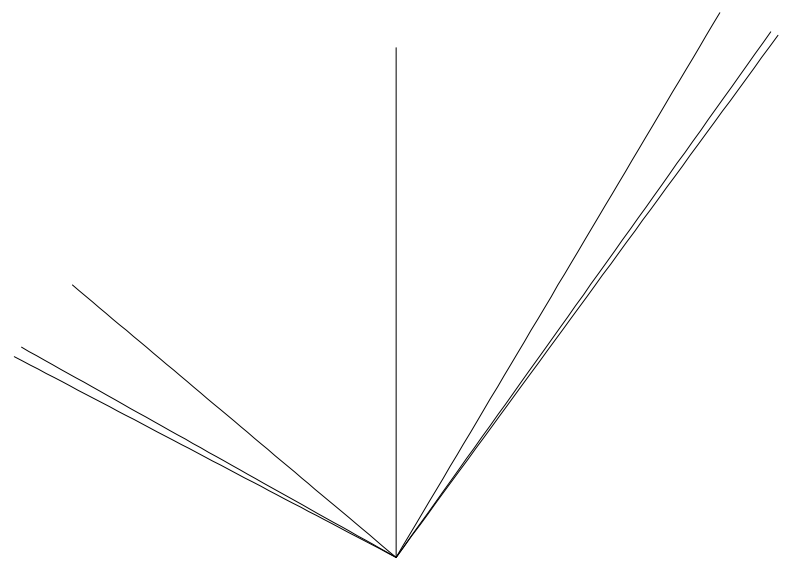

Figure 1. Decomposing the cone $\frac{2}{1-\sqrt{5}} y<x<\frac{2}{1+\sqrt{5}} y$.

However, when we include the action of $\operatorname{Aut}\left(X_{\mathcal{J}}\right)$ in our analysis, it may become possible to do the blowdowns - an infinite number of large radius limit points may turn into a finite

\footnotetext{
${ }^{10}$ The figure does not include these walls - the limiting rays with irrational slope $\frac{1 \pm \sqrt{5}}{2}$ - since they are less than a line-width's distance from the outer rays as shown (at the level of resolution of the figure).
} 
number after these identifications [54, 32]. In the example above, an automorphism acting on the cone as $(x, y) \mapsto(2 x+3 y, 3 x+5 y)$ leads from an infinite number of large radius limit points on the original Kähler moduli space to two remaining points on the quotient space. There are two boundary divisors (after taking the quotient), and they meet in two large radius limit points. The quotient space can then be blown down explicitly using methods of Hirzebruch [36], leading to a surface singularity with local equation $w^{2}=\left(u^{3}-v^{2}\right)\left(u^{2}-v^{3}\right)$. This is illustrated in figure 2 . In general, $\Xi$ will be a subset of the compactified moduli space only after taking such a quotient, which is why we use a local system $\mathcal{L}$ rather than simply a trivial sheaf.

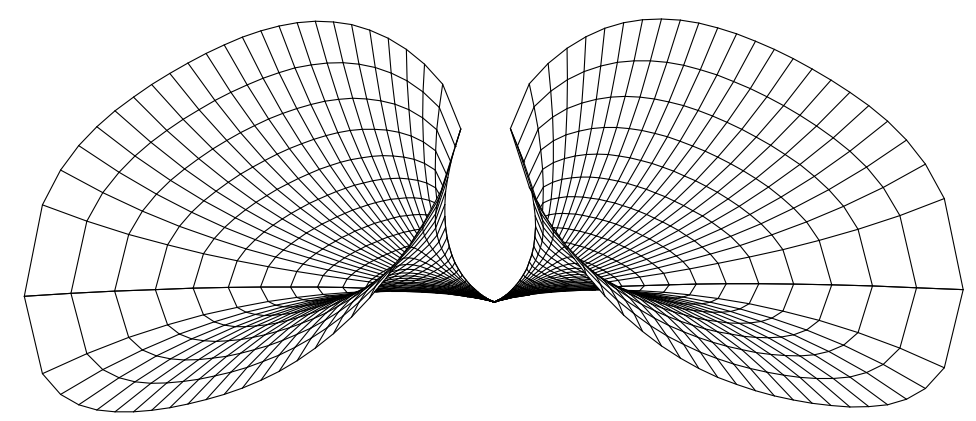

Figure 2. The blown down moduli space $w^{2}=\left(u^{3}-v^{2}\right)\left(u^{2}-v^{3}\right)$

Applying these ideas to the analysis of the B-model moduli space, we see that we should consider two large radius limit points to be equivalent when there exists a connected subset $\Xi$ of the boundary containing both points and a local system $\mathcal{L}$ defined in a neighborhood of $\Xi$ which is spanned by the canonical 1-forms $d \log z_{k}$ at any maximally unipotent point within $\Xi$. This can be effectively computed if we know the mirror map at each maximally unipotent point; in fact, it is enough to calculate the leading terms in Laurent expansions of 3 -point functions. Further details are in [54].

\section{Determining the mirror map (two conjectures)}

As pointed out in section 3, the most delicate part of determining the mirror map is specifying the constants of integration, passing from the canonical 1-forms $d \log z_{j}$ to the actual multi-valued coordinates $z_{j}$ themselves. There are two conjectures which have been used to determine these constants. The first one (stated here in detail for the first time) is completely general, applying in principle to all Calabi-Yau threefolds, while the second one is special to the case of toric hypersurfaces.

In addition to specifying the coordinates, one would like to specify a particular choice of holomorphic 3-forms $\Omega(z)$ in order to determine the 3-point functions precisely. This is something which the conjectures will also do. 


\subsection{A conjecture about integral cohomology}

The first conjecture for determining the mirror map involves the integral cohomology groups of a Calabi-Yau threefold. The conjecture is quite natural, and there is a bit of evidence for it in a few specific examples. We will give additional evidence here.

Simply put, we conjecture that the canonical coordinates and canonical gauge for $\Omega(z)$ should be given by periods over integer-valued cohomology classes, in the following precise sense. The classes $g^{0}, g^{1}, \ldots, g^{r}$ should be chosen from $H^{3}(Y, \mathbb{Z})$, in such a way that $g^{0}$ spans $W_{0} \cap H^{3}(Y, \mathbb{Z})$ and the entire set $g^{0}, g^{1}, \ldots, g^{r}$ spans $W_{2} \cap H^{3}(Y, \mathbb{Z})$. We conjecture that if this is done, and if we write $N^{(j)} g^{k}=m^{j k} G^{(0)}$ as in the previous section, then the matrix $\left(m^{j k}\right)$ is invertible over the integers. If this is true, then the mirror map will be uniquely specified by using the integral periods, and the gauge $\Omega(z)$ for which $\left\langle g^{0} \mid \Omega(z)\right\rangle= \pm 1$ will be uniquely specified (up to sign) as well. (This is because in any change of basis $g^{k} \mapsto \sum_{\ell=0}^{k} c_{\ell}^{k} g^{\ell}$ preserving the integral structure, we will have $c_{0}^{0}= \pm 1$ and $c_{0}^{k} \in \mathbb{Z}$ so that $\exp \left(2 \pi i\left(c_{0}^{k} / c_{0}^{0}\right)\right)=1$. $)$

Our conjecture is motivated by the observation that the integral structure on $H^{2}(X)$ controls the choice of coordinates there. In fact, the action of $N^{(j)}$ on $H^{3}(Y)$ can be seen as mirroring the action "cup product with $e^{j}$ " on $H^{*}(X)$, where $e^{j} \in H^{2}(X)$ is an integral class, part of the basis determining the coordinates. The idea that the integral structure on $H^{3}$ should mirror the integral structure on $H^{0} \oplus H^{2} \oplus H^{4} \oplus H^{6}$ is not a new one - it was explicitly mentioned in [4], for example, and it was used implicitly in the calculations of [19] (cf. also [17]). There is not a lot of evidence for this equivalence, however, other than the examples which we describe here.

As a practical matter, our conjecture can be tested in the following way. Compute the monodromy matrices $T^{(j)}$ with respect to a basis of integral cohomology. There must then be a rank one matrix $M$ (with image $W_{0}$ ) such that $N^{(j)} N^{(k)} N^{(\ell)}=c^{j k \ell} M$ for all $j, k, \ell$, where $c^{j k \ell}:=\left.e^{j} \cup e^{k} \cup e^{\ell}\right|_{[X]}$ are the intersection numbers on the mirror partner $Y$ of $X$. The conjecture states that $M$ should be a primitive integral matrix. ${ }^{11}$

The fact that the mirror map can perhaps be completely determined by looking at the integral structure was pointed out in [53], where it was shown that this conjecture holds for the case of the quintic-mirror (using calculations from [19]), and that the integral basis leads to the correct mirror map. In the two-parameter examples of [18] the same principle was used to determine the mirror map, with equal success. ${ }^{12}$ We will give additional evidence for our conjecture by verifying it (and checking that it produces the "correct" mirror map)

\footnotetext{
${ }^{11}$ In this version of testing the conjecture, it is assumed that a mirror partner is known. One could test the conjecture without this assumption by finding the primitive integral matrix $M$ first, calculating the corresponding coefficients $c^{j k \ell}$, and checking to see if they have the numerical properties compatible with Poincaré duality over the integers.

${ }^{12}$ The rank one matrices in those papers - denoted by $Y$ in eq. (7.4) of [18] and also by $Y$ preceding eq. (6.4) in [20] - have the property of being primitive integral matrices, although this was not pointed out in those papers.
} 
in the three further one-parameter examples studied in [52, 30, 43].

We must repeat the verification made in Appendix $\mathrm{C}$ of [53] that the monodromy of the actual period functions has a certain form. In fact, we will find a somewhat better normalization of that form this time. We will use the explicit monodromy calculations from [43]; a similar calculation could be done using [30] (which uses a different normalization of the parameter, making it difficult to compare to the present approach).

Our verification is displayed in table 1 . We show in the second column of the table the monodromy matrix $A$ as calculated in [43]. That matrix was not quite uniquely specified by the data with which those authors were working. In particular, there is freedom to replace $A$ by $A^{\prime}=m^{\prime} A\left(m^{\prime}\right)^{-1}$ for any matrix $m^{\prime} \in \operatorname{Sp}(4, \mathbb{Z})$ whose second and fourth rows are the same as that of the identity matrix. We make a choice of $m^{\prime}$, shown in the third column of the table, and calculate $A^{\prime}$ in the fourth column. Notice that the result takes the form

$$
A^{\prime}=\left[\begin{array}{rrcc}
1 & -1 & 0 & 1 \\
0 & 1 & 0 & -1 \\
-\lambda & 0 & 1 & 0 \\
-\lambda & \mu & 1 & 1-\mu
\end{array}\right]
$$

where $(\lambda, \mu)$ are as given in the fifth column of the table.

With $A^{\prime}$ in the given form, we can calculate the monodromy around infinity as

$$
T_{\infty}:=T^{-1} A^{\prime-1}=\left[\begin{array}{rrrr}
1 & 1 & 0 & 0 \\
0 & 1 & 0 & 0 \\
\lambda & \lambda & 1 & 0 \\
0 & -\mu & -1 & 1
\end{array}\right]
$$

where

$$
T:=\left[\begin{array}{cccc}
1 & 0 & 0 & 0 \\
0 & 1 & 0 & 1 \\
0 & 0 & 1 & 0 \\
0 & 0 & 0 & 1
\end{array}\right]
$$

We can thus easily see that $T_{\infty}$ is unipotent, with $\left(T_{\infty}-I\right)^{4}=0$.

It is then a straightforward computation to see that

$$
\left(-\log T_{\infty}\right)^{3}=\left[\begin{array}{cccc}
0 & 0 & 0 & 0 \\
0 & 0 & 0 & 0 \\
0 & 0 & 0 & 0 \\
0 & \lambda & 0 & 0
\end{array}\right] .
$$

Note that $\lambda$ is precisely the triple-self-intersection of an integral generator of $H^{2}$ of the mirror partner, verifying the conjecture in these cases. 


\begin{tabular}{|c|c|c|c|c|c|c|c|c|c|c|c|c|c|}
\hline$k$ & \multicolumn{4}{|c|}{$A$} & \multicolumn{4}{|c|}{$m^{\prime}$} & \multicolumn{4}{|c|}{$A^{\prime}=m^{\prime} A\left(m^{\prime}\right)^{-1}$} & $(\lambda, \mu)$ \\
\hline 5 & {$\left[\begin{array}{c}-9 \\
0 \\
-20 \\
-15\end{array}\right.$} & $\begin{array}{r}-3 \\
1 \\
-5 \\
5\end{array}$ & $\begin{array}{c}5 \\
0 \\
11 \\
8\end{array}$ & $\begin{array}{r}3 \\
-1 \\
5 \\
-4\end{array}$ & $\begin{array}{r}2 \\
0 \\
-5 \\
0\end{array}$ & $\begin{array}{l}0 \\
1 \\
0 \\
0\end{array}$ & $\begin{array}{r}-1 \\
0 \\
3 \\
0\end{array}$ & $\left.\begin{array}{l}0 \\
0 \\
0 \\
1\end{array}\right]$ & $\begin{array}{r}1 \\
0 \\
-5 \\
-5\end{array}$ & $\begin{array}{r}-1 \\
1 \\
0 \\
5\end{array}$ & $\begin{array}{l}0 \\
0 \\
1 \\
1\end{array}$ & $\left.\begin{array}{r}1 \\
-1 \\
0 \\
-4\end{array}\right]$ & $(5,5)$ \\
\hline 6 & $\begin{array}{r}1 \\
0 \\
-3 \\
-6\end{array}$ & $\begin{array}{r}-1 \\
1 \\
-3 \\
4\end{array}$ & $\begin{array}{l}0 \\
0 \\
1 \\
1\end{array}$ & $\begin{array}{r}1 \\
-1 \\
3 \\
-3\end{array}$ & {$\left[\begin{array}{r}1 \\
0 \\
-3 \\
0\end{array}\right.$} & $\begin{array}{l}0 \\
1 \\
0 \\
0\end{array}$ & $\begin{array}{l}0 \\
0 \\
1 \\
0\end{array}$ & $\left.\begin{array}{l}0 \\
0 \\
0 \\
1\end{array}\right]$ & {$\left[\begin{array}{r}1 \\
0 \\
-3 \\
-3\end{array}\right.$} & $\begin{array}{r}-1 \\
1 \\
0 \\
4\end{array}$ & $\begin{array}{l}0 \\
0 \\
1 \\
1\end{array}$ & $\left.\begin{array}{r}1 \\
-1 \\
0 \\
-3\end{array}\right]$ & $(3,4)$ \\
\hline 8 & $\begin{array}{r}1 \\
0 \\
-2 \\
-4\end{array}$ & $\begin{array}{r}-1 \\
1 \\
-2 \\
4\end{array}$ & $\begin{array}{l}0 \\
0 \\
1 \\
1\end{array}$ & $\begin{array}{r}1 \\
-1 \\
2 \\
-3\end{array}$ & {$\left[\begin{array}{r}1 \\
0 \\
-2 \\
0\end{array}\right.$} & $\begin{array}{l}0 \\
1 \\
0 \\
0\end{array}$ & $\begin{array}{l}0 \\
0 \\
1 \\
0\end{array}$ & $\left.\begin{array}{l}0 \\
0 \\
0 \\
1\end{array}\right]$ & {$\left[\begin{array}{r}1 \\
0 \\
-2 \\
-2\end{array}\right.$} & $\begin{array}{r}-1 \\
1 \\
0 \\
4\end{array}$ & $\begin{array}{l}0 \\
0 \\
1 \\
1\end{array}$ & $\left.\begin{array}{r}1 \\
-1 \\
0 \\
-3\end{array}\right]$ & $(2,4)$ \\
\hline 10 & $\begin{array}{l}1 \\
0 \\
0 \\
1\end{array}$ & $\begin{array}{l}0 \\
1 \\
1 \\
3\end{array}$ & $\begin{array}{l}1 \\
0 \\
1 \\
1\end{array}$ & $\begin{array}{r}0 \\
-1 \\
-1 \\
-2\end{array}$ & {$\left[\begin{array}{l}0 \\
0 \\
1 \\
0\end{array}\right.$} & $\begin{array}{l}0 \\
1 \\
0 \\
0\end{array}$ & $\begin{array}{r}-1 \\
0 \\
0 \\
0\end{array}$ & $\left.\begin{array}{l}0 \\
0 \\
0 \\
1\end{array}\right]$ & {$\left[\begin{array}{r}1 \\
0 \\
-1 \\
-1\end{array}\right.$} & $\begin{array}{r}-1 \\
1 \\
0 \\
3\end{array}$ & $\begin{array}{l}0 \\
0 \\
1 \\
1\end{array}$ & $\left.\begin{array}{r}1 \\
-1 \\
0 \\
-2\end{array}\right]$ & $(1,3)$ \\
\hline
\end{tabular}

Table 1: Monodromy calculations 


\subsection{The monomial-divisor mirror map}

In the case of toric hypersurfaces, there is an alternate conjectural method for specifying the mirror map, proposed in $[1],{ }^{13}$ and used with great success in $[2,3,37]$ (see also [18, 20]). Briefly, the parameters on both the A-model and B-model sides can be described by remarkably similar combinatorics; this similarity is used to write a conjecture for the derivative of the mirror map, which specifies the constants of integration. The conjecture was extended in [57] to also specify the "algebraic gauge" which should be used as a starting point for determining the natural gauge $\Omega(z)$; in addition, much evidence was amassed in [57] in favor of this approach. We refer the reader to [1] and [57] for details.

\section{Making enumerative predictions}

We are finally ready to put together all of the ingredients and describe the process of making enumerative predictions. The things which we are going to predict are the "numbers of rational curves" on a Calabi-Yau threefold, in the precise form of the "Gromov-Witten invariants" of the threefold. A mathematical version of these invariants has been extensively investigated [51,58] using Gromov's symplectic geometry techniques [35] which had inspired Witten's original work on the invariants [65, 66]. (An alternate proposed definition purely within algebraic geometry is currently under development by Kontsevich and Manin [46, 45].)

The steps in an enumerative prediction are these: given a proposed mirror pair $(X, Y)$, find the moduli space of complex structures on $Y$, blow up to obtain a model in which the boundary is a divisor with normal crossings, find the boundary points with maximally unipotent monodromy, and sort them into equivalence classes (as indicated in section 4). For one representative of each equivalence class, find the canonical coordinates $z_{j}$ and the canonical gauge $\Omega(z)$ (these are unique if the integral monodromy conjecture holds, otherwise make a choice), calculate the three-point functions

$$
\left\langle\frac{\partial}{\partial z_{j}} \frac{\partial}{\partial z_{k}} \frac{\partial}{\partial z_{\ell}}\right\rangle:=\int_{Y} \Omega(z) \wedge \nabla_{z_{j}} \nabla_{z_{k}} \nabla_{z_{\ell}} \Omega(z)
$$

in those coordinates and that gauge, and make a power series expansion

$$
(2 \pi i)^{3} z_{j} z_{k} z_{\ell} \int_{Y} \Omega(z) \wedge \nabla_{z_{j}} \nabla_{z_{k}} \nabla_{z_{\ell}} \Omega(z)=c^{j k \ell}+\sum_{\eta \in H_{2}} c_{\eta}^{j k \ell} z^{\eta},
$$

where the leading term $c^{j k \ell}$ should coincide with the intersection numbers on the mirror partner, and where we use a kind of multi-index notation for monomials $z^{\eta}$.

\footnotetext{
${ }^{13}$ Some signs were left unspecified in [1]. The proposal for determining the signs given in [3] is now in doubt; an alternate proposal [57] has much evidence in its favor.
} 
The coefficients $c_{\eta}^{j k \ell}$ themselves are not quite the predictions for "numbers of rational curves." One must take into account the "multiple cover formula" for the A-model $[5,50]^{14}$ and write the three-point functions in the form

$$
(2 \pi i)^{3} z_{j} z_{k} z_{\ell} \int_{Y} \Omega(z) \wedge \nabla_{z_{j}} \nabla_{z_{k}} \nabla_{z_{\ell}} \Omega(z)=c^{j k \ell}+\sum_{\eta \in H_{2}} \eta_{j} \eta_{k} \eta_{\ell} \varphi_{\eta} \frac{z^{\eta}}{1-z^{\eta}} .
$$

The coefficients $\varphi_{\eta}$ are then the predicted number of rational curves in the homology class $\eta$.

\section{References}

[1] P. S. Aspinwall, B. R. Greene, and D. R. Morrison, The monomial-divisor mirror map, Internat. Math. Res. Notices (1993), 319-337.

[2] __ Calabi-Yau moduli space, mirror manifolds and spacetime topology change in string theory, Nuclear Phys. B 416 (1994), 414-480.

[3] _ Measuring small distances in N=2 sigma models, Nuclear Phys. B 420 (1994), $184-242$.

[4] P. S. Aspinwall and C. A. Lütken, Quantum algebraic geometry of superstring compactifications, Nuclear Phys. B B355 (1991), 482-510.

[5] P. S. Aspinwall and D. R. Morrison, Topological field theory and rational curves, Comm. Math. Phys. 151 (1993), 245-262.

[6] Chiral rings do not suffice: $N=(2,2)$ theories with nonzero fundamental group, Phys. Lett. B 334 (1994), 79-86.

[7] _ Stable singularities in string theory (with an appendix by M. Gross), Cornell preprint CLNS-95-1325, 1995.

[8] V. V. Batyrev, Variations of the mixed Hodge structure of affine hypersurfaces in algebraic tori, Duke Math. J. 69 (1993), 349-409.

[9] — Quantum cohomology rings of toric manifolds, Journées de Géométrie Algébrique d'Orsay (Juillet 1992), Astérisque, vol. 218, Société Mathématique de France, 1993, pp. 9-34.

\footnotetext{
${ }^{14}$ Although this multiple cover formula - first postulated in [19] — can be derived using path integral arguments in conformal field theory [5] and has been mathematically proved for the algebraic Gromov-Witten invariants [50], it is still not known for the symplectic Gromov-Witten invariants.
} 
[10] V. V. Batyrev and D. van Straten, Generalized hypergeometric functions and rational curves on Calabi-Yau complete intersections in toric varieties, Comm. Math. Phys., to appear.

[11] P. Berglund, P. Candelas, X. de la Ossa, A. Font, T. Hübsch, D. Jančić, and F. Quevedo, Periods for Calabi-Yau and Landau-Ginzburg vacua, Nuclear Phys. B 419 (1994), 352403.

[12] P. Berglund and S. Katz, Mirror symmetry for hypersurfaces in weighted projective space and topological couplings, Nuclear Phys. B 420 (1994), 289-314.

[13] M. Bershadsky, S. Cecotti, H. Ooguri, and C. Vafa, Holomorphic anomalies in topological field theories (with an appendix by S. Katz), Nuclear Phys. B 405 (1993), 279-304.

[14] _ Kodaira-Spencer theory of gravity and exact results for quantum string amplitudes, Comm. Math. Phys. 165 (1994), 311-427.

[15] F. A. Bogomolov, Hamiltonian Kähler manifolds, Dokl. Akad. Nauk SSSR 243 (1978), no. 5, 1101-1104, English translation: Soviet Math. Dokl. 19 (1978), 1462-1465.

[16] R. L. Bryant and P. A. Griffiths, Some observations on the infinitesimal period relations for regular threefolds with trivial canonical bundle, Arithmetic and Geometry, vol. II, Geometry (M. Artin and J. Tate, eds.), Birkhäuser, Boston, 1983, pp. 77-102.

[17] P. Candelas and X. de la Ossa, Moduli space of Calabi-Yau manifolds, Nuclear Phys. B 355 (1991), 455-481.

[18] P. Candelas, X. de la Ossa, A. Font, S. Katz, and D. R. Morrison, Mirror symmetry for two parameter models (I), Nuclear Phys. B 416 (1994), 481-562.

[19] P. Candelas, X. C. de la Ossa, P. S. Green, and L. Parkes, A pair of Calabi-Yau manifolds as an exactly soluble superconformal theory, Nuclear Phys. B 359 (1991), 21-74.

[20] P. Candelas, A. Font, S. Katz, and D. R. Morrison, Mirror symmetry for two parameter models - II, Nuclear Phys. B 429 (1994), 626-674.

[21] P. Candelas, M. Lynker, and R. Schimmrigk, Calabi-Yau manifolds in weighted $\mathbb{P}_{4}$, Nuclear Phys. B 341 (1990), 383-402.

[22] E. Cattani and A. Kaplan, Polarized mixed Hodge structures and the local monodromy of a variation of Hodge structure, Invent. Math. 67 (1982), 101-115. 
[23] A. Ceresole, R. D'Auria, S. Ferrara, W. Lerche, J. Louis, and T. Regge, Picard-Fuchs equations, special geometry and target space duality, Essays on Mirror Manifolds, II, (B. R. Greene and S.-T. Yau, eds.), to appear.

[24] P. Deligne, Equations différentielles à points singuliers réguliers, Lecture Notes in Math., vol. 163, Springer-Verlag, Berlin, Heidelberg, New York, 1970.

[25] _ La conjecture de Weil, II, Inst. Hautes Études Sci. Publ. Math. 52 (1980), $137-252$.

[26] _ Local behavior of Hodge structures at infinity, Essays on Mirror Manifolds, II, (B. R. Greene and S.-T. Yau, eds.), to appear.

[27] R. Dijkgraaf and E. Witten, Topological gauge theories and group cohomology, Comm. Math. Phys. 129 (1990), 393-429.

[28] L. J. Dixon, Some world-sheet properties of superstring compactifications, on orbifolds and otherwise, Superstrings, Unified Theories, and Cosmology 1987 (G. Furlan et al., eds.), World Scientific, Singapore, New Jersey, Hong Kong, 1988, pp. 67-126.

[29] G. Ellingsrud and S. A. Strømme, The number of twisted cubic curves on the general quintic threefold, Math. Scand., to appear.

[30] A. Font, Periods and duality symmetries in Calabi-Yau compactifications, Nuclear Phys. B 391 (1993), 358-388.

[31] I. M. Gel'fand, A. V. Zelevinskiir and M. M. Kapranov, Hypergeometric functions and toral manifolds, Funktsional. Anal. i Prilozhen. 23 (1989), no. 2, 12-26, English translation: Functional Anal. Appl. 23 (1989), 94-106.

[32] A. Grassi and D. R. Morrison, Automorphisms and the Kähler cone of certain CalabiYau manifolds, Duke Math. J. 71 (1993), 831-838.

[33] B. R. Greene, D. R. Morrison, and M. R. Plesser, Mirror manifolds in higher dimension, Comm. Math. Phys., to appear.

[34] B. R. Greene and M. R. Plesser, Duality in Calabi-Yau moduli space, Nuclear Phys. B 338 (1990), 15-37.

[35] M. Gromov, Pseudo holomorphic curves in symplectic manifolds, Invent. Math. 82 (1985), 307-347.

[36] F. Hirzebruch, The ring of Hilbert modular forms for real quadratic fields of small discriminant, Modular Functions of One Variable VI (J.-P. Serre and D. B. Zagier, eds.), Lecture Notes in Math., vol. 627, Springer-Verlag, Berlin, Heidelberg, New York, 1977, pp. 287-323. 
[37] S. Hosono, A. Klemm, S. Theisen, and S.-T. Yau, Mirror symmetry, mirror map and applications to Calabi-Yau hypersurfaces, Comm. Math. Phys. 167 (1995), 301-350.

[38] - Mirror symmetry, mirror map and applications to complete intersection CalabiYau spaces, Nuclear Phys. B 433 (1995), 501-554.

[39] Y. Kanter, Computation of Yukawa couplings for Calabi-Yau hypersurfaces in weighted projective spaces, Phys. Lett. B 338 (1994), 204-211.

[40] N. M. Katz and T. Oda, On the differentiation of De Rham cohomology classes with respect to parameters, J. Math. Kyoto Univ. 8 (1968), 199-213.

[41] S. Katz, On the finiteness of rational curves on quintic threefolds, Compositio Math. 60 (1986), 151-162.

[42] _ Rational curves on Calabi-Yau manifolds: Verifying predictions of mirror symmetry, Projective Geometry with Applications (E. Ballico, ed.), Marcel Dekker, 1994, pp. 231-239.

[43] A. Klemm and S. Theisen, Considerations of one modulus Calabi-Yau compactifications: Picard-Fuchs equations, Kähler potentials and mirror maps, Nuclear Phys. B 389 (1993), 153-180.

[44] _ Mirror maps and instanton sums for complete intersections in weighted projective space, Mod. Phys. Lett. A 9 (1994), 1807-1818.

[45] M. Kontsevich, Enumeration of rational curves via torus actions, Max-Planck-Institut preprint MPI/94-39, 1994.

[46] M. Kontsevich and Yu. Manin, Gromov-Witten classes, quantum cohomology, and enumerative geometry, Comm. Math. Phys. 164 (1994), 525-562.

[47] A. Landman, On the Picard-Lefschetz transformations, Trans. Amer. Math. Soc. 181 (1973), 89-126.

[48] W. Lerche, C. Vafa, and N. P. Warner, Chiral rings in N=2 superconformal theories, Nuclear Phys. B 324 (1989), 427-474.

[49] A. Libgober and J. Teitelbaum, Lines on Calabi-Yau complete intersections, mirror symmetry, and Picard-Fuchs equations, Internat. Math. Res. Notices (1993), 29-39.

[50] Yu. I Manin, Generating functions in algebraic geometry and sums over trees, MaxPlanck-Institut preprint, 1994. 
[51] D. McDuff and D. Salamon, J-holomorphic curves and quantum cohomology, University Lecture Series, vol. 6, American Mathematical Society, Providence, 1994.

[52] D. R. Morrison, Picard-Fuchs equations and mirror maps for hypersurfaces, Essays on Mirror Manifolds (S.-T. Yau, ed.), International Press, Hong Kong, 1992, pp. 241-264.

[53] _ Mirror symmetry and rational curves on quintic threefolds: A guide for mathematicians, J. Amer. Math. Soc. 6 (1993), 223-247.

[54] — Compactifications of moduli spaces inspired by mirror symmetry, Journées de Géométrie Algébrique d'Orsay (Juillet 1992), Astérisque, vol. 218, Société Mathématique de France, 1993, pp. 243-271.

[55] — Where is the large radius limit?, Proceedings of Strings '93 (Berkeley), to appear.

[56] _ Mirror symmetry and moduli spaces of superconformal field theories, Proc. Intern. Congr. Math. Zurich, 1994, to appear.

[57] D. R. Morrison and M. R. Plesser, Summing the instantons: Quantum cohomology and mirror symmetry in toric varieties, Nuclear Phys. B, to appear.

[58] Y. Ruan and G. Tian, A mathematical theory of quantum cohomology, Math. Res. Lett. 1 (1994), 269-278.

[59] W. Schmid, Variation of Hodge structure: the singularities of the period mapping, Invent. Math. 22 (1973), 211-319.

[60] G. Tian, Smoothness of the universal deformation space of compact Calabi-Yau manifolds and its Peterson-Weil metric, Mathematical Aspects of String Theory (S.-T. Yau, ed.), World Scientific, Singapore, 1987, pp. 629-646.

[61] A. N. Todorov, The Weil-Petersson geometry of the moduli space of $S U(n \geq 3)$ (CalabiYau) manifolds, I, Comm. Math. Phys. 126 (1989), 325-246.

[62] C. Vafa, Modular invariance and discrete torsion on orbifolds, Nuclear Phys. B 273 (1986), 592-606.

[63] E. Viehweg, Weak positivity and the stability of certain Hilbert points, III, Invent. Math. 101 (1990), 521-543.

[64] C. Voisin, Miroirs et involutions sur les surfaces K3, Journées de Géométrie Algébrique d'Orsay (Juillet 1992), Astérisque, vol. 218, Société Mathématique de France, 1993, pp. 273-323. 
[65] E. Witten, Topological sigma models, Comm. Math. Phys. 118 (1988), 411-449.

[66] - On the structure of the topological phase of two dimensional gravity, Nuclear Phys. B 340 (1990), 281-332.

[67] _ Mirror manifolds and topological field theory, Essays on Mirror Manifolds (S.-T. Yau, ed.), International Press, 1992, pp. 120-159.

[68] _ Phases of $N=2$ theories in two dimensions, Nuclear Phys. B 403 (1993), 159222.

Department of Mathematics, Box 90320, Duke University, Durham, NC 27708-0320

E-mail address: drm@math.duke.edu 